\title{
Amyloid-ק-Derived Diffusible Ligands Cause Impaired Axonal Transport of Mitochondria in Neurons
}

\author{
Xinglong Wang ${ }^{a}$ George Perry a,b Mark A. Smith ${ }^{a}$ Xiongwei Zhu ${ }^{a}$ \\ a Department of Pathology, Case Western Reserve University, Cleveland, Ohio, and \\ ${ }^{b}$ College of Sciences, University of Texas at San Antonio, San Antonio, Tex., USA
}

\section{Key Words}

A $\beta$-derived diffusible ligands $\cdot$ Mitochondria $\cdot$ Axonal

transport $\cdot$ Alzheimer's disease

\begin{abstract}
Background: Alzheimer's disease (AD) is the most prevalent form of dementia predominantly affecting the elderly. It is believed that soluble amyloid- $\beta(A \beta)$ oligomers are involved in the pathogenesis of $A D$, yet the underlying mechanisms remain elusive. Objectives: Emerging evidence suggests that mitochondrial dysfunction likely plays a critical role in $A \beta$-induced neuronal degeneration. Previously, we demonstrated that $A \beta$-derived diffusible ligands (ADDLs) induce reduced mitochondrial density in neurites, and we suspect that an impaired mitochondrial trafficking might be involved, which is tested in this study. Methods: Using live cell imaging, anterograde and retrograde transport of mitochondria in primary hippocampal neurons treated with sublethal doses of ADDLs was measured. Results: We found that ADDLs induced significant impairment in both anterograde and retrograde transport of mitochondria along axons. Conclusion: These results suggest that an impaired mitochon-
\end{abstract}

drial transport likely contributes to ADDL-induced abnormal mitochondrial distribution and dysfunction and also reinforce the idea that axonal transport is likely involved in $A D$ pathogenesis.

Copyright $\odot 2010$ S. Karger AG, Basel

\section{Introduction}

Alzheimer's disease (AD) is the most common neurodegenerative disorder in the aged population and is characterized by two pathological hallmarks, i.e., senile plaques and neurofibrillary tangles, along with extensive neuronal death in selected brain regions [1]. Despite extensive research efforts, the mechanisms underlying neurodegeneration in $\mathrm{AD}$ remain elusive. Largely due to the fact that $A D$-causing mutations found in amyloid- $\beta(A \beta)$ precursor protein (APP) and presenilin 1 and 2 have a primary effect on $A \beta$ processing and plaque formation, a myriad of studies have focused on the central role of $A \beta$ in the pathogenesis and progression of $\mathrm{AD}$, and it is generally believed that the soluble oligomers of $A \beta$ are the major toxic species. Emerging evidence suggest that mi- 
Fig. 1. Effect of ADDLs on FAT of mitochondria. Rat E18 hippocampal neurons (DIV 9) were transfected with MitoDsRed2. $24 \mathrm{~h}$ after incubation with or without $800 \mathrm{~nm}$ ADDLs at DIV 11, neurons were imaged in time-lapse (10 s interval, $20 \mathrm{~min}$ ). a Representative kymographs showing transport of mitochondria in the segment of axon around $100 \mu \mathrm{m}$ in length beginning $300 \mu \mathrm{m}$ from the cell body of control neurons or neurons treated with A $342-1$ and ADDLs. b Quantification of mitochondria flux (number mitochondria/10 $\mathrm{min}$ ) revealed that both anterograde and retrograde FAT of mitochondria were greatly impaired by ADDLs rather than negative control or $A \beta 42-1$. At least 20 neurons were analyzed in three independent experiments $\left({ }^{*} \mathrm{p}<0.05\right.$, Student's t test).

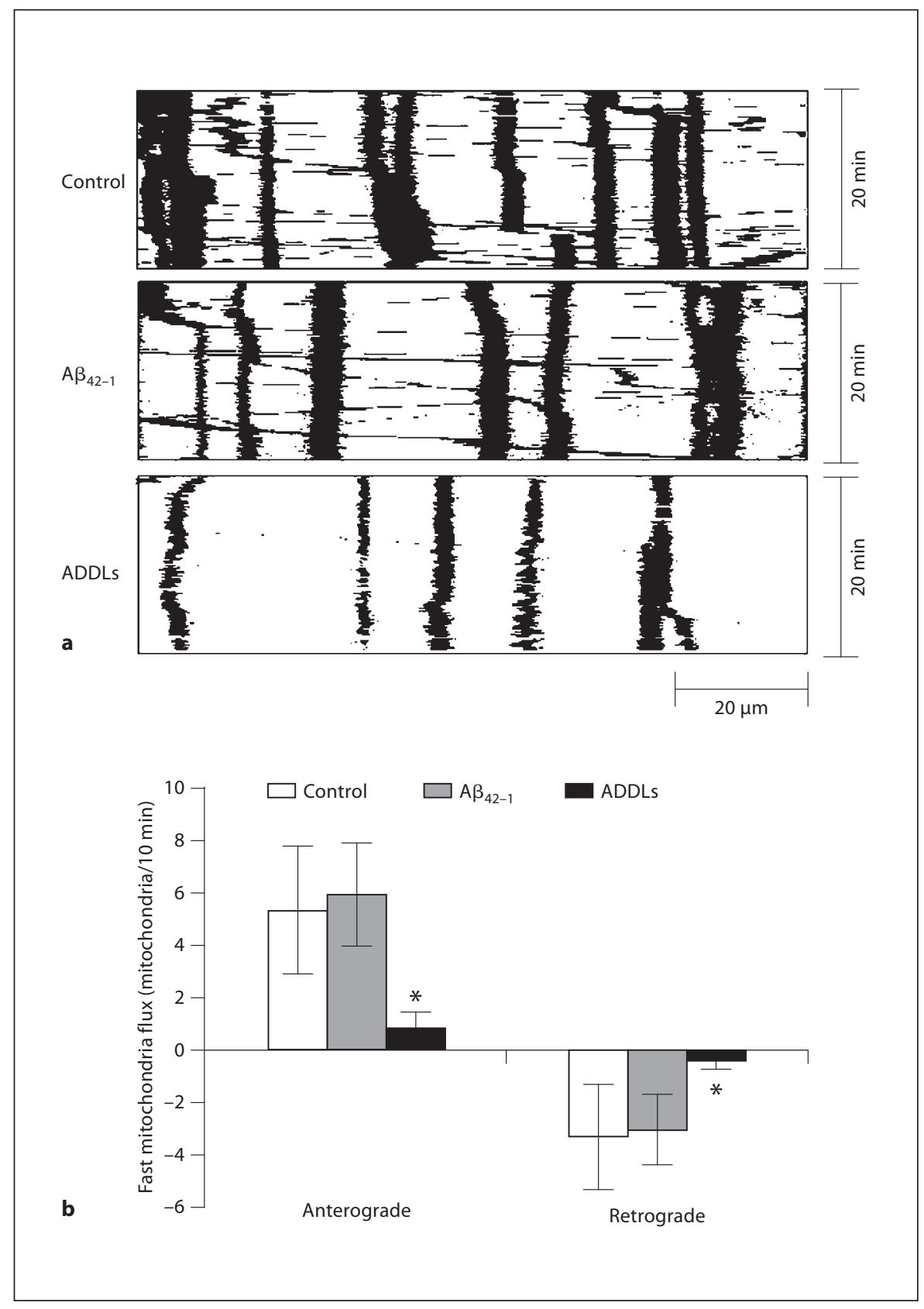

tochondria are a major intracellular target of soluble $A \beta$ oligomers [2]. Interestingly, we found abnormal mitochondrial dynamics in $\mathrm{AD}$ fibroblasts [3] and demonstrated that APP overexpression, likely through A $\beta$ production, induced abnormal mitochondrial dynamics in neuronal cells [4]. Most recently, we confirmed abnormal mitochondrial dynamics in $\mathrm{AD}$ neurons and demonstrated that $A \beta$-derived diffusible ligand (ADDLs) in- duced abnormal mitochondrial dynamics in neurons [5]. One of the common features in all these models demonstrating abnormal mitochondrial dynamics is an abnormal mitochondrial distribution, i.e., perinuclear accumulation of mitochondria in AD fibroblasts or M17 cells overexpressing mutant APP or reduced mitochondrial density in neurites of primary hippocampal neurons. Since fast axonal transport (FAT) of mitochondria un- 
derlies the uniform distribution of mitochondria along the axon [6], these findings prompted us to question whether an abnormal mitochondrial transport is involved. In this study, by using live cell imaging, we investigated axonal transport in ADDL-treated primary hippocampal neurons.

\section{Materials and Methods}

\section{Cell Culture and Transfection}

Primary neurons from E18 rat hippocampus (BrainBits) were seeded at 30,000-40,000 cells per well on $35 \times 10 \mathrm{~mm}$ culture dishes coated with poly-D-lysine/laminin in neurobasal medium supplemented with 2\% B27 (Invitrogen)/0.5 mM glutamine/25 $\mathrm{mm}$ glutamate. Half the culture medium was changed every 3 days with neurobasal medium supplemented with 2\% B27 (Invitrogen) and $0.5 \mathrm{~mm}$ glutamine. All cultures were kept at $37^{\circ} \mathrm{C}$ in a humidified $5 \% \mathrm{CO}_{2}$-containing atmosphere. More than $90 \%$ of cells were neurons after they were cultured for 12 days in vitro (DIV), verified by positive staining for neuronal specific markers microtubule-associated protein-2 (MAP2, dendritic marker) and Tau-1 (axonal marker). At DIV 9, neurons were transfected with Mito-DsRed 2 construct (Clontech) using Neurofect (Genlantis) according to the manufacturer's protocol.

Time-Lapse Imaging and Image Analysis

ADDLs were prepared as previously described [5]. Two days after transfection with Mito-DsRed2, neurons were treated with ADDLs and then put in a well-equipped environmental chamber with controlled $\mathrm{CO}_{2}$ content, humidity and temperature. Timelapse images were captured with an inverted Leica DMI6000 fluorescence microscope (Leica) with a fully automated scanning stage and Leica EL6000 alignment-free metal halide bulb. The microscope was driven by MetaMorph software (Version 7.04, Molecular Devices). Images were acquired using a $\times 401.24 \mathrm{NA}$ objective and a Retiga-EXi CCD digital camera (QImaging). Positive transfected neurons were identified by their expression of red fluorescence, and three different neurons were selected for time-lapse imaging at the same time. Images were acquired every $10 \mathrm{~s}$ for a total of 121 images $(20 \mathrm{~min})$ without apparent phototoxicity or photobleaching. Kymographs were generated using MetaMorph software (Molecular Devices). Image analysis was performed with WCIF ImageJ (developed by W. Rasband) and MetaMorph software (Molecular Devices).

\section{Results}

To investigate FAT of mitochondria, rat E18 hippocampal neurons were plated on poly-D-lysine/laminincoated glass dishes and transfected with Mito-DsRed2 to label mitochondria at DIV $9.48 \mathrm{~h}$ after transfection, neurons were treated with $800 \mathrm{nM}$ ADDLs or $10 \mu \mathrm{M}$ A $342-1.24 \mathrm{~h}$ after treatment, mitochondria movement in neurons was imaged for up to $20 \mathrm{~min}$ under fluores- cent microscope for time-lapse recordings. No cell death was observed under these conditions as determined by $\mathrm{LDH}$ assay (not shown). Because of high mitochondria density in the proximal segment of axon, we just measured FAT of mitochondria in the relative distal segment of axon (around $100 \mu \mathrm{m}$ in length beginning 300 $\mu \mathrm{m}$ from the cell body of neurons; fig. 1a). Consistent with our prior report [5], ADDL treatment led to less mitochondria in axons. FAT of mitochondria included anterograde and retrograde movements, and mitochondria with velocity less than $0.1 \mu \mathrm{m} / \mathrm{s}$ were classified as stationary. The mitochondria flux that passed a single point in the center of kymographs was counted as described before [7]. Notably, in ADDL-treated neurons, both anterograde $(0.80 \pm 0.67)$ and retrograde FAT $(0.44 \pm 0.21)$ of mitochondria were significantly reduced compared to nontreated control neurons (5.34 \pm 2.47 for anterograde and $3.31 \pm 2.02$ for retrograde transport of mitochondria; fig. 1b). Neurons treated with A $342-1$ demonstrated similar FAT of mitochondria to control neurons.

\section{Discussion}

Previously, it was demonstrated that acute treatment of $A \beta$ monomers and fibrils induces significant reduction in motile mitochondria [8]. In this study, we performed a more detailed study on axonal transport of mitochondria and found that soluble $A \beta$ oligomers induced significantly reduced mitochondrial axonal transport, suggesting that impaired axonal transport of mitochondria likely contributes to ADDL-induced abnormal mitochondrial distribution in neurites. This is consistent with a recent study demonstrating an overall disruption of FAT induced by soluble $A \beta$ oligomers in isolated squid axoplasms [9]. In fact, deficits in axonal transport may represent an early step in $\mathrm{AD}$ pathogenesis since axonal swelling and reduced axonal transport were observed before apparent AD hallmarks [10]. Overall, our studies, along with others, suggest that $A \beta$ is likely one of the major mediators causing deficits in axonal transport.

\section{Acknowledgements}

This study is supported by the NIH (AG 031852), Alzheimer's Association (IIRG-07-60196, Zen-07-59500) and a pilot grant from Memory and Cognition Center at Case Western Reserve University. 


\section{References}

1 Smith MA: Alzheimer disease. Int Rev Neurobiol 1998;42:1-54.

-2 Reddy PH: Amyloid beta, mitochondrial structural and functional dynamics in Alzheimer's disease. Exp Neurol 2009;218:286292.

-3 Wang X, Su B, Fujioka H, Zhu X: Dynaminlike protein 1 reduction underlies mitochondrial morphology and distribution abnormalities in fibroblasts from sporadic Alzheimer's disease patients. Am J Pathol 2008; 173:470-482.
4 Wang X, Su B, Siedlak SL, Moreira PI, Fujioka H, Wang Y, Casadesus G, Zhu X: Amyloid-beta overproduction causes abnormal mitochondrial dynamics via differential modulation of mitochondrial fission/fusion proteins. Proc Natl Acad Sci USA 2008; 105 19318-19323.

5 Wang X, Su B, Lee HG, Li X, Perry G, Smith MA, Zhu X: Impaired mitochondrial fission and fusion in Alzheimer's disease. J Neurosci 2009;29:9090-9103.

6 Hollenbeck PJ, Saxton WM: The axonal transport of mitochondria. J Cell Sci 2005; 118:5411-5419.

7 Miller KE, Sheetz MP: Axonal mitochondrial transport and potential are correlated. J Cell Sci 2004;117:2791-2804.
8 Rui Y, Tiwari P, Xie Z, Zheng JQ: Acute impairment of mitochondrial trafficking by beta-amyloid peptides in hippocampal neurons. J Neurosci 2006;26:10480-10487.

-9 Pigino G, Morfini G, Atagi Y, Deshpande A, Yu C, Jungbauer L, LaDu M, Busciglio J, Brady S: Disruption of fast axonal transport is a pathogenic mechanism for intraneuronal amyloid beta. Proc Natl Acad Sci USA 2009;106:5907-5912.

10 Stokin GB, Lillo C, Falzone TL, Brusch RG, Rockenstein E, Mount SL, Raman R, Davies P, Masliah E, Williams DS, Goldstein LS: Axonopathy and transport deficits early in the pathogenesis of Alzheimer's disease. Science 2005;307:1282-1288. 